\title{
Trends in Diabetic Ketoacidosis Presentations at a Major Rural Referral Centre in Australia
}

\author{
Dr Tamara Young - endocrinology Advanced Trainee, Orange Health Service (2014)
}

\section{OBJECTIVES}

To study trends in presentation and management of diabetic ketoacidosis (DKA) at a rural referral centre, to ultimately assist with development of a formal management protocol

\section{BACKGROUND}

Orange Health Service is a 520 bed campus with a 12 bed intensive care/ high dependency unit; the largest in central west NSW. The referral base is up to 34 outlying facilities. (4) Patients are transported from outlying facilities utilising NSW ambulance health service or specialist retrieval services via road. Patients are generally transferred to a facility such as Orange Health service with capacity for monitoring in a high dependency unit. (5). This unique demographic formed the background of our single centre study to examine trends in DKA presentation over a two year period.

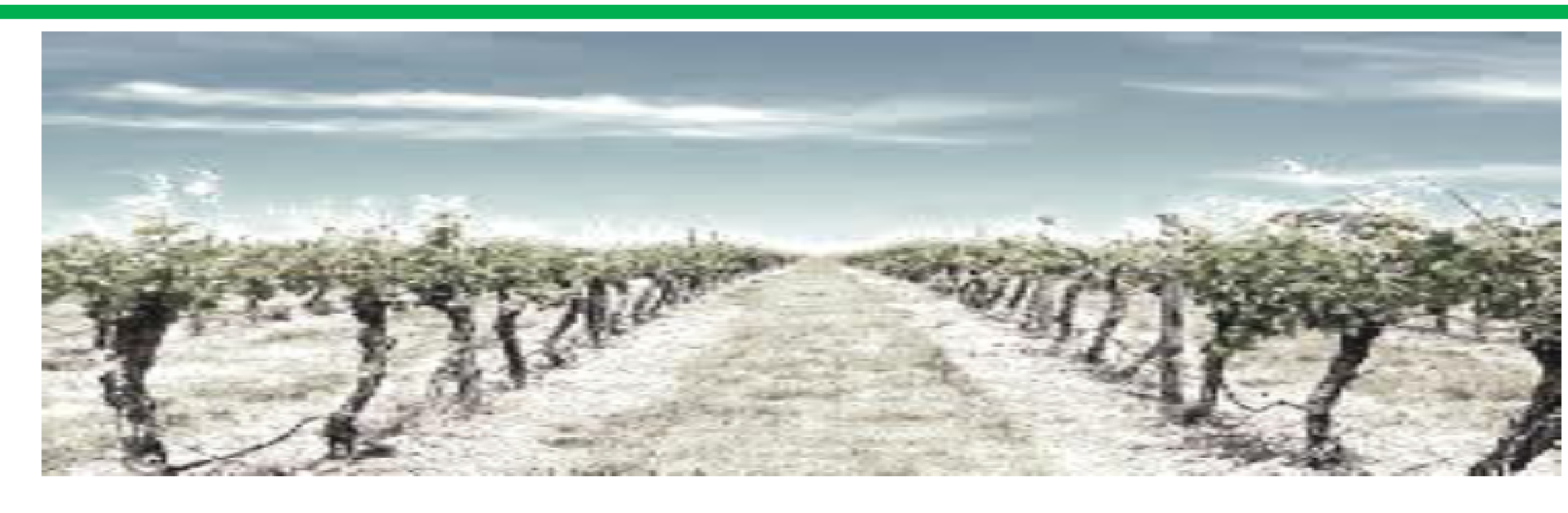

CONCLUSIONS

The implementation of a DKA protocol in a facility such as Orange Health Service has its own unique challenges Patients treated Orange Health Service for management of diabetic ketoacidosis have a tendency towards a severe presentation and many are transported over a long distance to our facility. When developing a protocol for appropriate management of diabetic ketoacidosis at a major rural hospital, multiple factors including the higher acuity of patient presentation and possibility of transfer from an outlying facility need to be considered that could potentially impact on initial and ongoing management.

\section{INTRODUCTION AND METHODS}

The study design was a retrospective chart review of two years of data. Ethical approval was sought from Western NSW Local Health District to access medical records.

A coding search was done for patients presenting with "diabetic ketoacidosis" as principal or other diagnosis over a 2 year period. A review of medical records was undertaken to determine if the clinical presentation was consistent with this diagnosis. The parameters used to define DKA (1) were 1) ketonemia of $3 \mathrm{mmol}$ or significant (defined as $2+$ ketonuria). 2) Blood glucose over $11 \mathrm{mmol}$ or known diabetes and 3) bicarbonate less than $15 \mathrm{mmol}$ and/or venous $\mathrm{pH}$ less than 7.3. Patients aged $<16$ years old at the time of presentation were also excluded.

Data sourced for all presentations of DKA was age of patient, distance from Orange Health Service, new onset versus known type 1 diabetes mellitus, initial pH, time to normalisation of ketones, any episodes of hypokalaemia or clinically significant hypotension during their management, episodes of hyperchloraemia, and overall length of stay. Statistical analysis was carried out to determine the mean and standard deviation, where data was normally distributed, and the median when data was not normally distributed.

Furthermore, Spearman's correlations were conducted to examine the relationship between distance and age, length of stay and initial pH.

\section{RESULTS}

23 cases met the inclusion criteria for analysis. The mean age of patients was 25 years. (standard deviation 8.1 years). The mean length of stay was 4.57 days (standard deviation of 3.34 days). Complications were recorded in half of patients with complete data sets. The mean initial pH was 7.134 with a standard deviation of 0.144 . The median distance was $5 \mathrm{~km}$, with $61 \%$ of cases based within Orange. The interquartile range was 126 kilometres. There was a statistically significant positive association between age and length of stay (rs $=0.44, n=23, p<0.05$ ) and a statistically significant negative association between length of stay and initial pH (rs = -0.51, $n=23, p<0.05)$. Distance was not correlated with length of stay nor initial $\mathbf{p H}$.
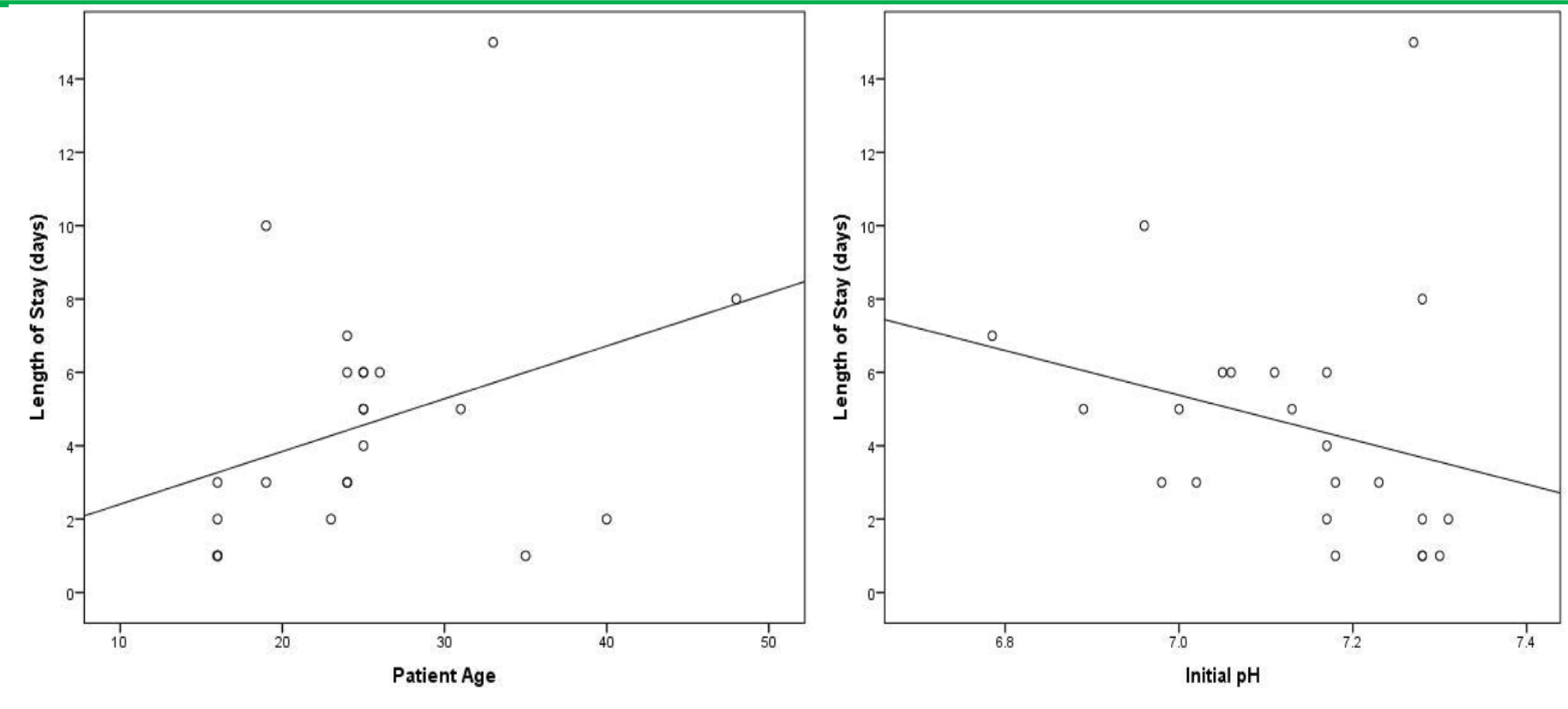

REFERENCES 